\title{
Direct comparison of ARPES, STM, and quantum oscillation data for band structure determination in $\mathrm{Sr}_{2} \mathrm{RhO}_{4}$
}

\author{
I. Battisti ${ }^{1}$, W. O. Tromp $\mathbb{D}^{1}$, S. Riccò ${ }^{2}$, R. S. Perry ${ }^{3,4}$, A. P. Mackenzie $\mathbb{D}^{5,6 凶}$, A. Tamai ${ }^{2}$, F. Baumberger $\mathbb{D}^{2,7 凶}$ and M. P. Allan $\mathbb{D}^{1 凶}$
}

Discrepancies in the low-energy quasiparticle dispersion extracted from angle-resolved photoemission, scanning tunneling spectroscopy, and quantum oscillation data are common and have long haunted the field of quantum matter physics. Here, we directly test the consistency of results from these three techniques by comparing data from the correlated $\mathrm{metal} \mathrm{Sr}_{2} \mathrm{RhO}_{4}$. Using established schemes for the interpretation of the experimental data, we find good agreement for the Fermi surface topography and carrier effective masses. Hence, the apparent absence of such an agreement in other quantum materials, including the cuprates, suggests that the electronic states in these materials are of different, non-Fermi liquid-like nature. Finally, we discuss the potential and challenges in extracting carrier lifetimes from photoemission and quasiparticle interference data.

npj Quantum Materials (2020)5:91; https://doi.org/10.1038/s41535-020-00292-4

\section{INTRODUCTION}

Strongly correlated electrons are at the root of some of the most mysterious quantum materials, including unconventional superconductors, strange metals, and heavy fermion materials ${ }^{1-5}$. Most of the exotic phases of electronic matter in these systems emerge from collective behavior of the electrons. A universally accepted understanding of these systems is still lacking, and requires close cooperation between scientists using different theoretical and experimental methods. From the experimental side, many insights to date have come from spectroscopic techniques that probe the band structure and many-body renormalizations of electrons close to the Fermi level, including angle-resolved photoemission (ARPES), scanning tunneling microscopy (STM), and quantum oscillations (QO), which are the focus of this article.

In the most widely used interpretations, spectroscopic-imaging STM (SI-STM) and ARPES probe the spectral function in real and reciprocal space ${ }^{6-10}$. Quantum oscillations probe the Fermi surface area and the $k$-averaged cyclotron mass which can in turn be related to the pole of the spectral function at energies close to the Fermi level ${ }^{11}$. There should thus be well-defined relations between the quantities measured by these three techniques ${ }^{12}$.

Surprisingly though, several apparent contradictions between results based on these techniques can be found in the literature. Such contradictions can involve very fundamental properties of the electronic structure: for example, quantum oscillation studies on underdoped cuprate high-temperature superconductors claim the existence of Fermi surface pockets while STM and ARPES reported disconnected Fermi arcs ${ }^{13,14}$. Similarly, the strength of gap inhomogeneities seen by STM in several unconventional superconductors appears to be inconsistent with gap broadening in ARPES spectra that average over large areas. These and other discrepancies between results of different techniques have previously been discussed in cuprate superconductors ${ }^{15-18}$, heavy fermion systems ${ }^{19}$, and topological insulators ${ }^{20}$. However, it often remains unknown if these apparent differences are a consequence of some inherent limitations of the techniques or if they are due to challenges of data interpretation, also connected to the exotic non-Fermi-liquid nature of some of these systems. Given this lack of understanding, discrepancies are frequently attributed to the use of samples grown in different research laboratories or are ignored because of a lack of trust in one of the techniques.

With this article, we aim to test the consistency of data from ARPES, STM, and QO experiments by making an unbiased comparison on the same correlated electron material. The ideal candidate for such a comparison should be a quasi-twodimensional (2D) metal in which electron correlations still play an important role, but without the mysteries associated with materials like unconventional superconductors. Ideally it should further be structurally similar to the cuprates, ruthenates, and iridates. Such a material could then act as a representative for the wider class of transition metal oxides, but in contrast to cuprates, ruthenates and iridates is well understood and simple enough that it can clearly be described within Fermi liquid theory. With this in mind, we chose $\mathrm{Sr}_{2} \mathrm{RhO}_{4}$, a layered perovskite that fulfills the conditions above.

\section{RESULTS \& DISCUSSIONS}

ARPES and QPI Fermi surface

In Figs 1 and 2, we compare Fermi surface data from the three techniques. Consistent with previous reports ${ }^{11,21,22}$, the ARPES $k$-space map (Fig. 1a) shows two nearly circular contours that are backfolded to form three pockets; a hole-like a pocket centered at $\Gamma$, a lens-shaped electron pocket at $M\left(\beta_{M}\right)$ and a square-shaped hole pocket at $X\left(\beta_{X}\right)$. The backfolding is of structural origin and arises from a staggered rotation of the $\mathrm{RhO}_{6}$ octahedra around the $c$-axis, which doubles the in-plane unit cell. Hybridization with $e_{g}$ states pushes the $x y$ band of $\mathrm{Sr}_{2} \mathrm{RhO}_{4}$ below the chemical potential, leaving a Fermi surface with out-of-plane $x z / y z$ character, containing three electrons per Rh site ${ }^{11,22,23}$. Despite the quasi-1D hopping associated with the out-of-plane orbitals, the Fermi surface is nearly isotropic. This change arises from a

\footnotetext{
${ }^{1}$ Leiden Institute of Physics, Leiden University, Niels Bohrweg 2, 2333 CA Leiden, The Netherlands. ${ }^{2}$ Department of Quantum Matter Physics, University of Geneva, 24 Quai ErnestAnsermet, 1211 Geneva, Switzerland. ${ }^{3}$ London Centre for Nanotechnology and UCL Centre for Materials Discovery, University College London, London WC1E 6BT, United Kingdom. ${ }^{4}$ ISIS Facility, Rutherford Appleton Laboratory, STFC, Chilton, Didcot OX11 0QX, United Kingdom. ${ }^{5}$ Max Planck Institute for Chemical Physics of Solids, 01187 Dresden,

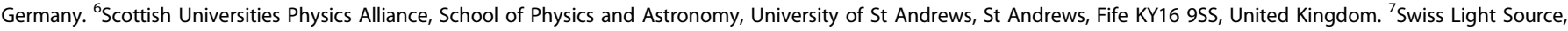
Paul Scherrer Institute, 5232 Villigen PSI, Switzerland. ${ }^{凶}$ email: Andy.Mackenzie@cpfs.mpg.de; Felix.Baumberger@unige.ch; allan@physics.leidenuniv.nl
} 
a

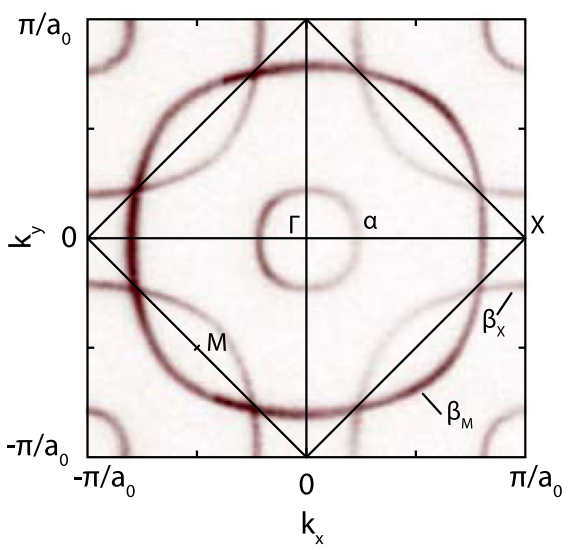

d

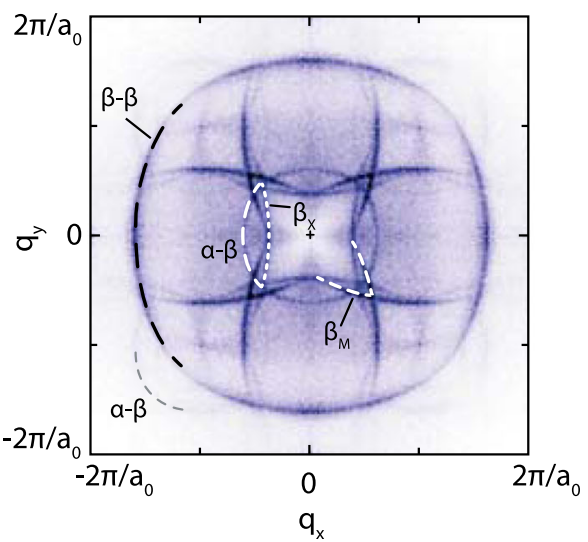

b

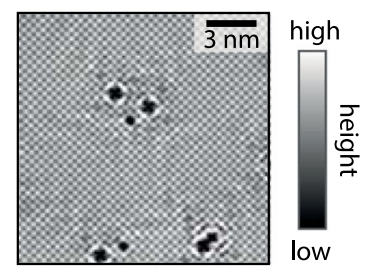

C

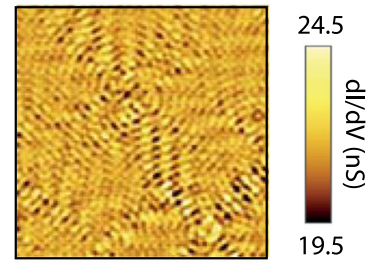

e

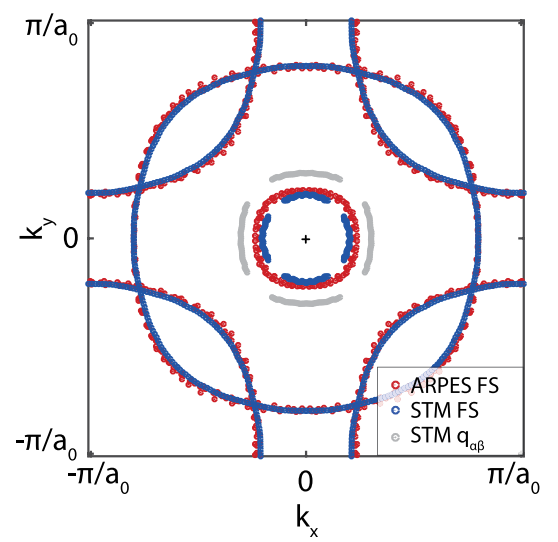

Fig. $1 \mathrm{Sr}_{2} \mathrm{RhO}_{4}$ Fermi surfaces. a ARPES Fermi surface. b STM topograph with atomic resolution showing presence of impurities. c STM conductance layer $d l / d V(r, e V)$ at energy $e V=-20 \mathrm{meV}$, acquired simultaneously to the topograph in panel $\mathbf{b}$, showing interference between quasiparticles standing waves patterns. d STM 'Fermi surface', obtained by a two-dimensional Fourier transform of the conductance layer corresponding to the Fermi level of a spectroscopic map measured over a field of view of $70 \times 70 \mathrm{~nm}^{2}$. Here and for all QPI data, we show the normalized data, i.e., the Fourier transform of $d l / d V(r, e V) /(I(\mathbf{r}) / \mathrm{eV})$, to mitigate the set-up effect (see Methods). The data is additionally symmetrized and the low-q components are suppressed with a 2D gaussian: raw data is shown in Supplementary Fig. 1. e Comparison between extracted Fermi surfaces of ARPES and STM. For completeness, we also show the QPI signal of $\mathbf{q}_{\alpha \beta}$, which was used in the derivation (see text and Supplementary Figs 2 and 3).

a

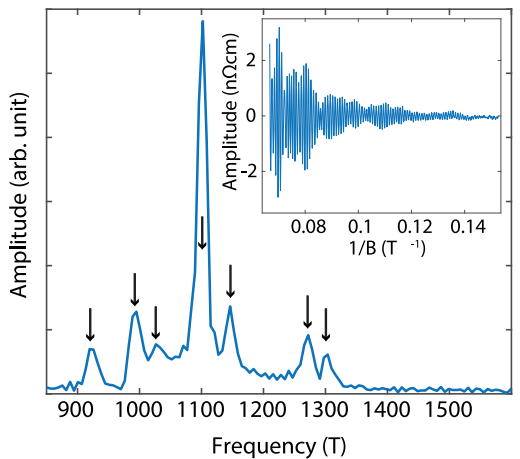

b

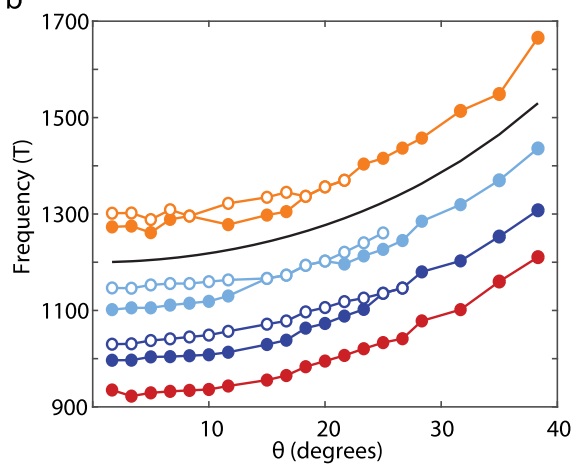

C $\quad E_{F}-0.02 \mathrm{eV}$

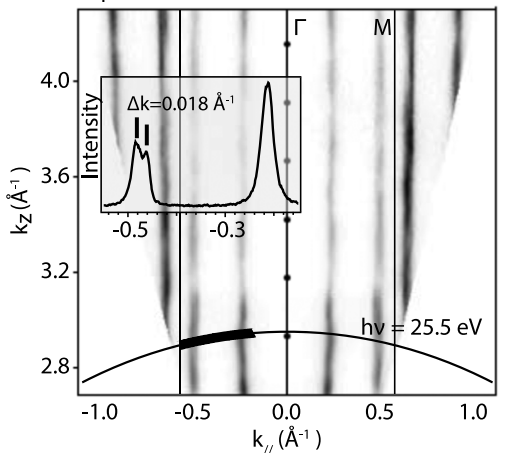

Fig. 2 Quantum oscillations and $\boldsymbol{k}_{\boldsymbol{z}}$ dispersion. a Shubnikov-de Haas oscillations at $0.1 \mathrm{~K}$ for a magnetic field parallel to the $c$-axis. The main panel shows the frequency components obtained by Fourier transform of the quantum oscillation trace shown in the inset. The peaks correspond to Fermi surface pockets. The background subtraction used was a third-order polynomial and the field sweep rate was $0.05 \mathrm{~T} / \mathrm{min}$. The noise level is $50 \mathrm{pVHz}^{-1 / 2}$. b Angle dependence of the QO frequencies for angle $\theta$ from the $c$-axis. The solid black line is a $1 / \cos \theta$ dependence expected for a quasi-two-dimensional Fermi surface. c ARPES $k_{z}$ dependence in the M $\Gamma$ high-symmetry direction at $E=-20 \mathrm{meV}$, showing only slight modulations of the band along the $c$-axis. 
strong level repulsion of states that would be degenerate in the absence of spin-orbit coupling ${ }^{24,25}$. The marked anticrossing can be attributed to an enhancement of spin-orbit splittings in the presence of electronic correlations ${ }^{25-27}$.

Figure $1 \mathrm{~b}, \mathrm{c}$ shows an STM topography and a constant energy conductance layer, where spatial modulations attributed to quasiparticle interference are neatly resolved. The few atomic defects in the field of view clearly act as scattering centers for quasiparticles, creating the interfering standing wave patterns. The Fourier transform of the normalized conductance layer at the Fermi level $E=0 \mathrm{meV}$ is shown in Fig. 1d. To mitigate the set-up effect, we take the Fourier transform not of the conductance layers $d l / d V(\mathbf{r}, e V)$, but of the normalized conductance data, $d l / d V(\mathbf{r}$, $\mathrm{eV}) /(I(\mathbf{r}, \mathrm{eV}) / \mathrm{V})$, where $I(\mathbf{r}, \mathrm{eV})$ is the tunneling current and $V$ is the bias voltage (see discussion in the methods and Supplementary Fig. 1$)^{28-32}$. For the $\beta$ band, we directly observe the STM 'Fermi surface' with wave-vector $\mathbf{q}=2 \mathbf{k}_{\mathrm{F}}$. More generally, we expect to observe features corresponding to scattering vectors $q$ that connect points of high spectral weight in momentum space. For the present case, we can readily connect these q vectors with the Fermi surface measurement from ARPES. Interestingly, different scattering processes have different strengths. While some scattering processes are very clear, others are less visible or completely absent. Varying intensities or absences of scattering processes have been observed in other materials $\mathrm{s}^{33-35}$ and can stem from the differences in the scattering process. For example, different QPI scattering intensities are expected from magnetic versus potential scattering or from broad coulombic potentials versus localized impurity potentials ${ }^{36-40}$. In principle, theoretical tools exist to predict QPI intensities based on both the electronic structure of the material and the nature of the scattering potential ${ }^{36-40}$. A comparison with such QPI simulations could allow us to learn more about the defect states in $\mathrm{Sr}_{2} \mathrm{RhO}_{4}$.

In Fig. 1e, we use the Fourier transform of the SI-STM data discussed above to reconstruct the entire $\mathrm{FS}$ of $\mathrm{Sr}_{2} \mathrm{RhO}_{4}$ from the QPI pattern. To this end, we first extract peaks in the data by fitting the intensity profiles in radial cuts (see Supplementary Fig. 2). We then obtain the fundamental $\beta$ band in $k$-space by rescaling the $q$ vectors by a factor of two. The backfolded $\beta$ bands are obtained by translating the fundamental band by reciprocal lattice vectors determined from the STM topography. The a band is reconstructed by subtracting the interband vector $\mathbf{q}_{\alpha-\beta}$ from the intraband scattering $\mathbf{q}_{\beta-\beta}$. In Fig. $1 e$, we display in blue the data points derived in this way, and in gray the direct interband scattering vectors $\mathbf{q}_{\alpha-\beta}$ that we used for the derivation. The identification of the gray scattering vectors as $\mathbf{q}_{a-\beta}$ is corroborated by the Fermi velocity we obtain for this vector (see below). The direct comparison of these contours with the ARPES Fermi surface shows good agreement for all Fermi surface pockets, as further illustrated in Supplementary Fig. 3.

\section{Shubnikov-de Haas Oscillations}

Next, we use QO to find the Fermi surface areas and quasiparticle masses. The inset of Fig. 2a shows a trace of quantum oscillations in the magnetoresistance at $0.1 \mathrm{~K}$ (Shubnikov-de Haas $(\mathrm{SdH})$ oscillations). To extract Fermi surface information from the quantum oscillations measurement, we analyze the frequency components plotted in Fig. 2a. Seven closely spaced peaks are resolved, corresponding to seven frequencies between 0.9 and $1.3 \mathrm{kT}$. This might be surprising at first, as from our previous analysis we expect only three distinct Fermi surface pockets. We attribute the higher number of $\mathrm{QO}$ frequencies to two effects. Firstly, the finite interlayer hopping implies that the Fermi surface of $\mathrm{Sr}_{2} \mathrm{RhO}_{4}$ is quasi-cylindrical, and thus has multiple extremal orbits per sheet. The characteristic signature of such a remnant 3D Fermi surface warping is an overall $1 / \cos (\theta)$ field angle dependence of the frequencies (consistent with quasi-2D electronic structure) with small splittings that disappear for certain angles, as observed in Fig. 2b. The quasi-2D nature of the quasiparticle band structure is confirmed directly by photon-energy-dependent ARPES measurements (Fig. 2c) probing the Fermi surface along $k_{z}$. Secondly, the ARPES measurements resolve a small splitting in the $\beta$-band along $Г \mathrm{M}$. This small degeneracy lifting can be attributed to the doubling of the unit cell along the $c$-axis and is reproduced by $\mathrm{LDA}+\mathrm{U}+\mathrm{SO}$ band structure calculations ${ }^{25}$. Hence, there are four primary frequencies up to the measured out-of-plane angle of $40^{\circ}$. We can then use multiple facts to constrain the band assignments: (i), Following ARPES and STM data, the extremal orbit areas increase in size from the $a\left(\right.$ hole), $\beta_{M}$ (electron), and $\beta_{x}$ (hole). (ii), The total electron count should be three electrons per Rh atom. (iii), The experimental specific heat $\gamma$ can be calculated in the $2 \mathrm{D}$ approximation from:

$\gamma=\left(\pi N_{\mathrm{A}} k_{\mathrm{B}}^{2} a^{2}\right)\left(3 \hbar^{2}\right)^{-1}\left(m_{\mathrm{a}}+2 m_{\beta \mathrm{M}}+m_{\beta \mathrm{X}}\right)$

where $N_{\mathrm{A}}$ is Avogadro's number, $k_{\mathrm{B}}$ is Boltzmann's constant, $a$ is the tetragonal lattice parameter $(3.857 \AA)$, and $\hbar$ is Planck's constant. (iv), Following the ARPES data, the $\beta_{M}$ band should be split leading to two frequencies.

Combining these conditions, we draw the conclusion that a corresponds to the lowest frequency $(0.93 \mathrm{kT}$, corresponding to 1.934 electron/Rh, see methods), $\beta_{M}$ to the two middle frequencies (average $1.068 \mathrm{kT}, 0.152$ electrons $/ \mathrm{Rh}$ ), and $\beta_{\mathrm{X}}$ to the highest frequency $(1.288 \mathrm{kT}, 0.908$ electrons/Rh). The calculated $\gamma=17.4 \pm$ $0.8 \mathrm{~mJ} / \mathrm{Rh} \mathrm{mol} \mathrm{K}^{2}$ then agrees with the directly observed value of $17.7 \pm 0.7 \mathrm{~mJ} / \mathrm{Rh} \mathrm{mol} \mathrm{K}^{222}$, and the total electron count is 2.994 electrons per Rh. A quantitative comparison of the Fermi surface volumes extracted from ARPES, QO, and STM based on this assignment can be found in Table 1. QO amplitudes also yield the Dingle temperatures $\left(1.5 \mathrm{~K}\right.$ for the a pocket, $1.5 \mathrm{~K}$ for the $\beta_{\mathrm{M}}$ pocket, and $1.9 \mathrm{~K}$ for the $\beta_{\mathrm{x}}$ pocket), which can be related to the mean free

Table 1. Summary of band structure parameters from different techniques.

\begin{tabular}{llll}
\hline & $\alpha$ & $\beta_{\mathrm{M}}$ & $\beta_{\mathrm{X}}$ \\
\hline$v_{F}(e V \AA)$ & & & \\
QO & $0.41^{*}$ & $0.47^{*}$ & $0.49^{*}$ \\
ARPES & 0.41 & $0.57 / 0.77$ & 0.55 \\
STM & $0.41^{*}$ & 0.70 & 0.55 \\
$<$ A $>$ (\%) & & & \\
ARPES & $7.7 \%$ & $7.9 \%$ & $9.1 \%$ \\
STM & $6.8 \%$ & $7.1 \%$ & $7.9 \%$ \\
QO $<$ peaks $>$ & $6.6 \%$ & $7.6 \%$ & $9.2 \%$ \\
QO & $6.6 \%$ & $7.1 \%$ & $9.1 \%$ \\
single peak & & $7.3 \%$ & $9.3 \%$ \\
& & $7.9 \%$ & \\
& & $8.2 \%$ & \\
$<>\left(m_{e}\right)$ & & & 3.1 \\
QO & 3.1 & 2.9 & 3.3 \\
ARPES & 3.3 & 2.2 & 2.9 \\
STM & - & 2.4 & \\
\hline
\end{tabular}

Comparison between values obtained from the three techniques. $v_{F}$ is the Fermi velocity, $A$ (in \% of the reduced tetragonal Brillouin zone) and $<m>$ (in units of $m_{e}$ ) are the Brillouin zone filling and average mass for each of the three sheets, respectively. The pocket-averaged Fermi velocities from QO (marked with ${ }^{*}$ ) are extracted using $\hbar k_{\mathrm{F}}=m v_{\mathrm{F}}$, using the pocketaveraged Fermi wave vector. The Fermi velocity for the $\alpha$ band from STM (also marked with ${ }^{*}$ ) was extracted from the slope of the $\mathbf{q}_{\alpha \beta}$ and $\mathbf{q}_{\beta \beta}$ signals. The QO values for $A$ are given both as average over the multiple peaks for each pocket, and for every peak. 

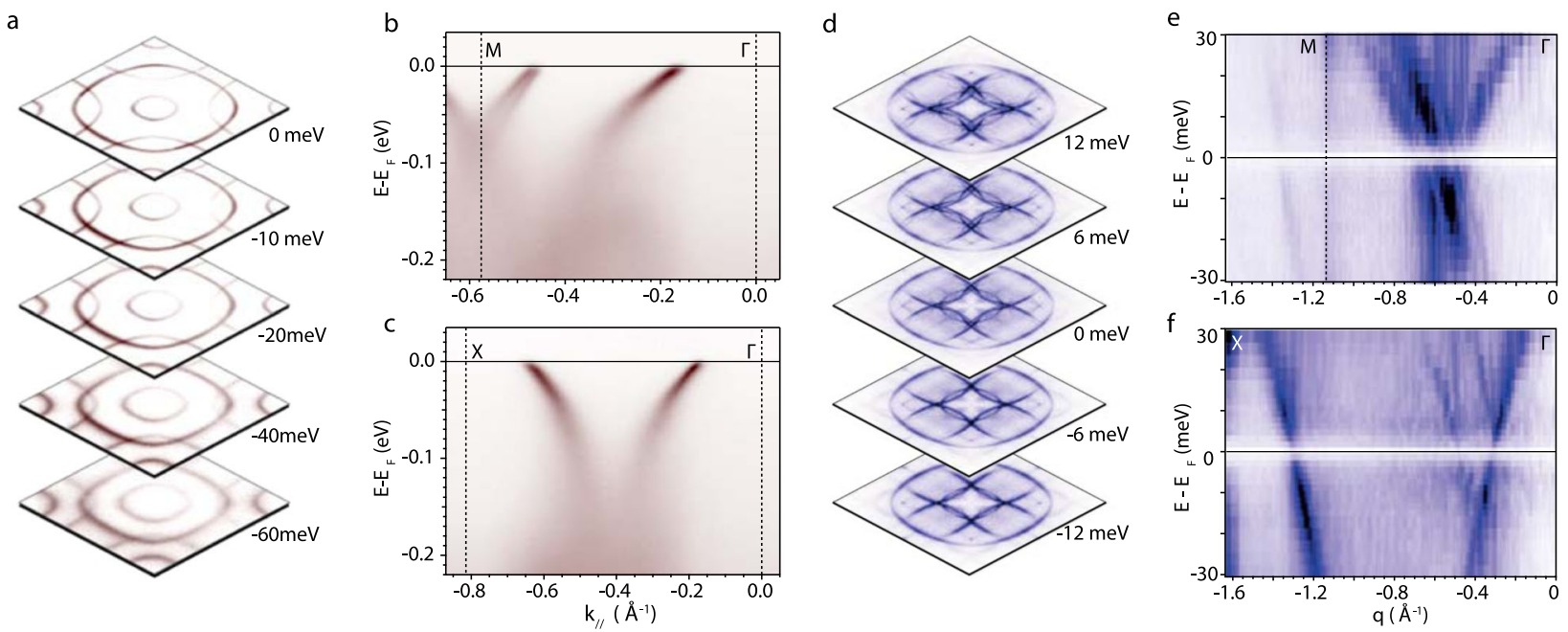

Fig. 3 ARPES and QPI dispersions along high-symmetry directions. a ARPES constant energy layers at selected energies at and below the Fermi level. b-c ARPES dispersions along the high-symmetry directions $M \Gamma$ and $X \Gamma$. $\mathbf{d}$ Fourier transform of normalized STM conductance layers $d l / d V(\mathbf{r}, e V) /(I(\mathbf{r}) / V)$ at selected energies around the Fermi level. e-f Dispersions of the scattering vectors $\mathbf{q}$ obtained from STM QPI for the two high-symmetry directions $\mathrm{M} \Gamma$ and $\mathrm{X} \Gamma$, where $\mathrm{X}$ and $\mathrm{M}$ are defined as for ARPES but at double the reciprocal vectors. To improve signal-tonoise, the dispersions are obtained by averaging 10 cuts in a radial span of $\pm 5^{\circ}$ from the high-symmetry direction. The intensity around the Fermi level is suppressed due to the normalization with the total conductance $(d l / d V) /(I / M)$, as explained in the Methods.

path of the electrons. We refer to the literature for a detailed discussion on the challenges of such an interpretation ${ }^{41}$.

\section{Quasiparticle dispersion}

We now turn our attention to the low-energy dispersion. In Fig. 3, we show constant energy layers for selected energy levels and the energy-momentum dispersion along the two high-symmetry directions for both ARPES and SI-STM. These data confirm that $\beta_{M}$ is an electron pocket while $a$ and $\beta_{X}$ are hole-like. The ARPES data also reproduce the splitting of the $\beta$ band along $\Gamma \mathrm{M}$ observed in Fig. 2. The STM dispersion plots show several features that are not observed by ARPES. These can all be assigned to different $\beta-\beta$ intraband and $\alpha-\beta$ interband scattering vectors translated by reciprocal lattice vectors. The $\mathbf{q}$ vector which is most clearly resolved by STM along both high-symmetry directions arises from $\beta-\beta$ intraband scattering. Comparing its dispersion with the $\beta$-band measured by ARPES, we find quantitative agreement along $\Gamma X$, where both techniques lead to measured Fermi velocities $v_{\mathrm{F}}=0.55 \mathrm{eVÅ}$. Along $\Gamma \mathrm{M}$, where band structure calculations find a small splitting in the Fermi surface, our ARPES data resolves both bands and shows that they have slightly different dispersion with Fermi velocities of 0.57 and $0.77 \mathrm{eVA}$, respectively, compared to $v_{\mathrm{F}}=0.70 \mathrm{eVÅ}$ extracted from the STM dispersion. The lack of a noticeable splitting in the STM dispersion cannot be explained by insufficient momentum resolution, suggesting that it is due to a vanishing STM matrix element for one of the bands.

In order to extend this comparison to QO, we look at the quasiparticle cyclotron masses $m^{*}$. These masses can be deduced for individual Fermi surface pockets from the temperature dependence of the quantum oscillation amplitudes using the Lifshitz-Kosevich formula (Supplementary Fig. 4). For a 2D Fermi surface, they can also be calculated without any approximations from the full mapping of the low-energy quasiparticle band structure obtained by STM and ARPES, using $m^{*}=\frac{\hbar^{2}}{2 \pi} \frac{\mathrm{d} A_{\mathrm{FS}}}{\mathrm{dE}}$ (2) where $A_{\mathrm{FS}}$ is the Fermi surface volume. To this end, we extract the areas of the pockets not only at the Fermi energy, but at a few constant energy layers within a small window. The linear fits of these areas shown in Fig. 4 yield the effective masses of the different pockets. We note that the slope $\mathrm{d} A_{\mathrm{FS}} / \mathrm{d} E$ decreases strongly near the chemical potential in the ARPES data while no such effect is

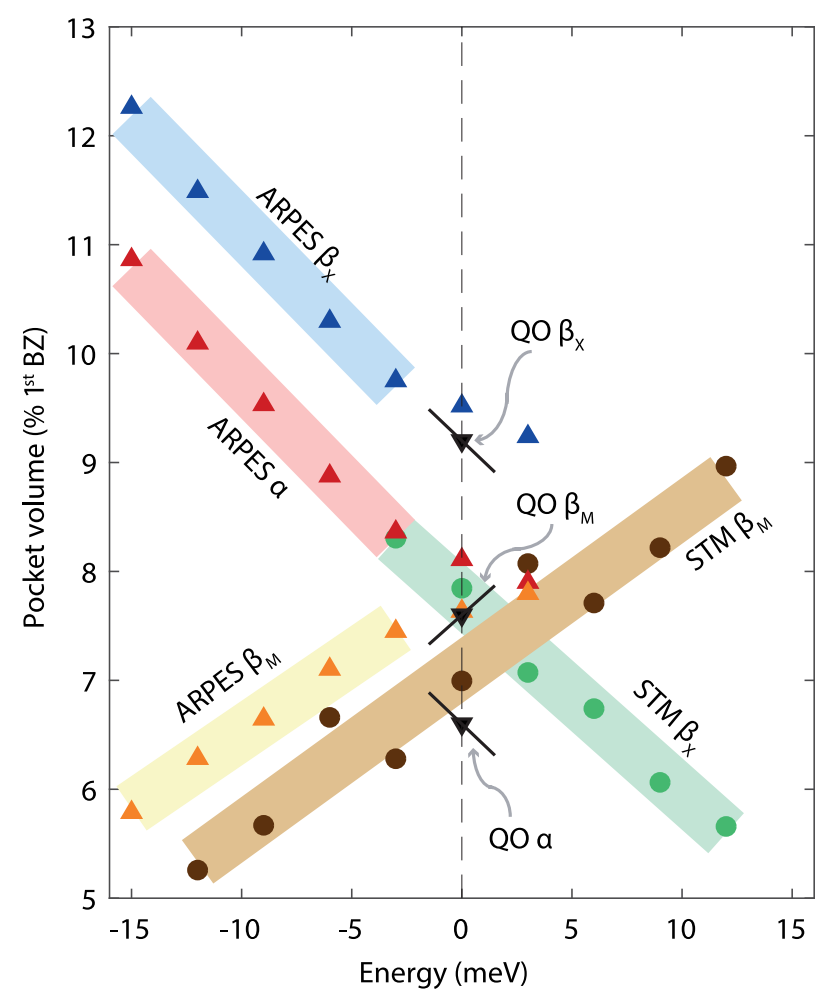

Fig. 4 Fermi surface pockets and effective masses. Extraction of effective masses from ARPES, STM, and QO. The data points show the volume $\mathrm{d} A_{\mathrm{FS}}(\omega)$ of the different pockets as a function of energy (see also Supplementary Fig. 5). The effective masses are proportional to the slope of $\mathrm{d} A_{\mathrm{FS}}(\omega)$. For the fits of the ARPES data, we excluded data points that are closer to the Fermi level than the energy resolution of the detector. The black lines and marks around the Fermi level indicate the masses and volumes extracted from QO.

observed in STM. This change of slope is a known artifact arising from the combination of a Fermi cutoff and finite energy resolution. For the quantification of $m^{*}$, we thus exclude a narrow energy range around $E_{\mathrm{F}}$ from the ARPES data. Table 1 shows the 
values of the effective masses obtained by STM, ARPES, and QO measurements. Knowledge of $m^{*}$ and the Fermi surface area also allows a sheet-averaged Fermi velocity to be calculated from QO data as $v_{\mathrm{F}}=\hbar(A / \pi)^{1 / 2} / m^{*}$ (3). These values are also shown in Table 1 for comparison with STM and ARPES.

\section{Lifetime analysis}

Finally, we discuss the extraction of peak widths in the ARPES and STM data, which can in principle be related to quasiparticle lifetimes. Here, the two techniques face rather different challenges. In simple systems, like $\mathrm{Sr}_{2} \mathrm{RhO}_{4}$ studied here, the measured photoemission intensity appears to represent the spectral function. For a sufficiently linear band, the imaginary part of the self-energy (which is equal to half the inverse lifetime in a Fermi liquid) can thus be obtained from the width $W_{k}$ of the momentum distribution curves (MDC) as $\Sigma^{\prime \prime}(k, \omega)=W_{k}(\omega) v(\omega) / 2(4)$, where $v(\omega)$ is the slope of the dispersion at the same energy. In practice, the main difficulty is the treatment of the effective resolution of ARPES measurement. ARPES peak widths measured at state-of-the-art instruments are rarely limited by the instrumental energy and momentum resolution but contain a variety of other contributions that are notoriously hard to quantify. These include in particular broadening from the finite integration over perpendicular momenta, structural mosaicity in the probed area and the oftenunknown quality of the surface. Additional broadening can occur from work function inhomogeneities of and around the sample which cause uncontrolled electric fields that degrade the resolution of the electron optics. Finally, in an energy range of $\sim \mathrm{d} E / 2$, where $\mathrm{d} E$ is the effective energy resolution, the MDC peak position starts to deviate noticeably from the intrinsic quasiparticle pole, which prohibits a model-free analysis of very lowenergy dispersions and self-energies. STM does not suffer from these experimental difficulties. However, it is not always clear to what extent the tunneling spectra reflect $A(\mathbf{r}, \omega)$. In particular the so-called set-up effect, the dependence of the tunneling spectra on the lateral variation of the tip-sample distance, which itself is defined by the set-up current and voltage, can cause complications. In many cases, the set-up effect can be mitigated by taking ratios between different quantities as we do here, but then the interpretation of the resulting data is less straight forward. In addition, analyzing self-energies from STM data can be complicated when different $\mathbf{q}$ vectors overlap, especially for complex Fermi surfaces. We also note that a unique reconstruction of the spectral function from STM data is not always possible. Perhaps most importantly, one has to consider the scattering mechanism, which can strongly influence line-shapes and linewidths ${ }^{36-40}$. For this reason, only few attempts have been made to extract lifetimes from STM data ${ }^{42-45}$

Despite these difficulties, an analysis of the MDC's along the $\Gamma X$ direction shown in Fig. 5a-c clearly shows an energy dependence of the quasiparticle lifetime in fair agreement with the expectations for a Fermi liquid-like metal. In a Fermi liquid, we expect the imaginary part of the self-energy to be a quadratic function at low energy, $\Sigma^{\prime \prime}(\omega)=A \omega^{2}(5)$, where $A$ is a material specific prefactor. To compare our results with the expectation, we plot the linewidths and self-energies extracted from ARPES and STM data as a function of energy in Fig. $5 \mathrm{~d}$, e (for a comparison of the individual ARPES and STM spectral lines, see Supplementary Fig. 6). Indeed, our results are consistent with a quadratic dependence on energy. Further, the absolute scale of the measured self-energy is of the same order than what is obtained for $\mathrm{Sr}_{2} \mathrm{RhO}_{4}$ with dynamical mean field theory (DMFT) ${ }^{46,47}$ calculated for a generic twodimensional Fermi liquid using the random phase approximation $^{48-51}$. We note that the agreement holds for an energy range that is a significant fraction of the Fermi energy, which is roughly $400 \mathrm{meV}$ for the $\beta$ band when defined in the parabolic band approximation as $E_{\mathrm{F}}=\hbar^{2} A_{\mathrm{FS}} / 2 \pi m$ (6). This is encouraging for further lifetime investigations: given a good understanding of both the resolution for ARPES and the scattering process for STM, both techniques have the potential to bring insight into energyand momentum-dependent correlation effects in electronic matter of quantum materials. a

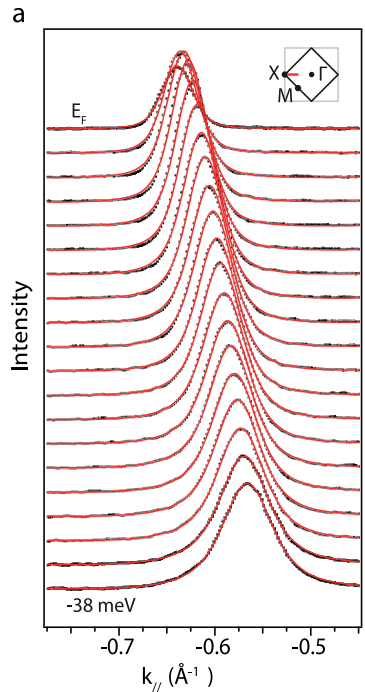

b

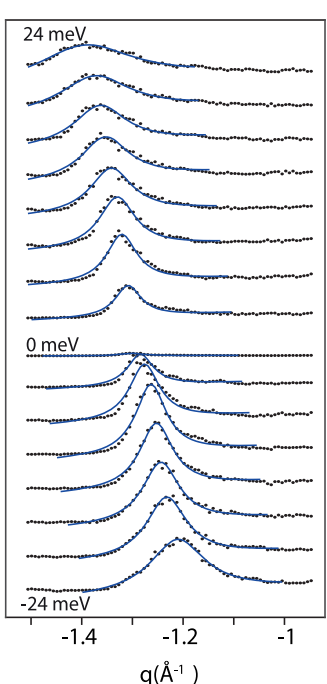

c

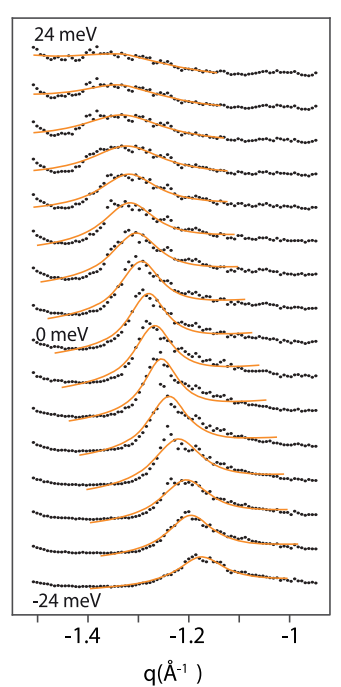

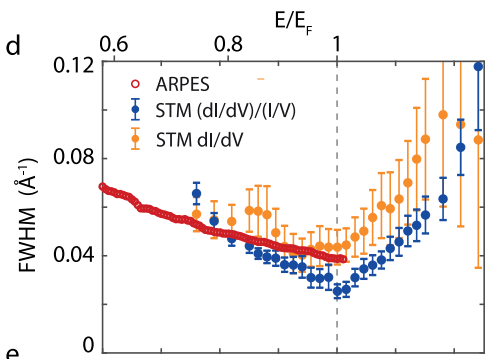

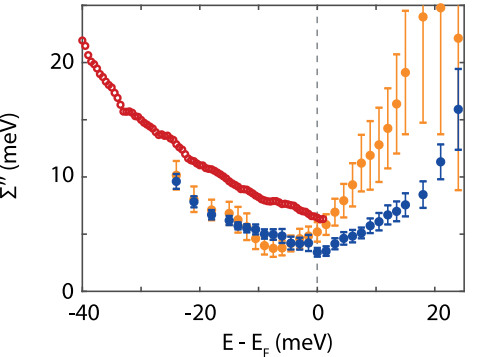

Fig. 5 ARPES and QPI linewidths and self-energies. Linewidth analysis for the $\beta_{X}$ band. a ARPES MDCs and Lorentzian fits (red). The inset shows the position of the cut as red line in the reduced Brillouin zone (black square). b, c STM MDCs from normalized conductance ( $d / / d V) /(I / M)$ (panel b) and conductance $d I / d V$ (panel c) including Lorentzian fits (blue and orange, respectively) with a linear background. The cuts are equivalent to the one used in panel a at double the reciprocal lattice vectors. d Comparison of the MDC widths $W_{k}(\omega)$ from STM and ARPES data. STM widths are phenomenological full-width-half-maximum extracted by Lorentzian fits, ARPES widths are extracted by fitting a Lorentzian convoluted with a Gaussian broadening that stems from the finite resolution. e Comparison of the widths multiplied by the slope of the dispersion, which, in a simplified picture, equals to the imaginary part of the self-energy. Note that the scale of the energy axis spans a significant fraction of the Fermi energy, which is roughly $400 \mathrm{meV}$ for the $\beta$ band (in the parabolic band approximation and before hybridization, as we assume that scattering processes that are relevant for the lifetimes do not discriminate between the $\beta_{X}$ and $\beta_{M}$ bands). 
We show SI-STM results of $\mathrm{Sr}_{2} \mathrm{RhO}_{4}$ and extract its Fermi surface and low-energy dispersion, and present a quantitative comparison of the STM quasiparticle interference data with ARPES and QOs. Our data here reveals the previously unknown band structure above the Fermi level, and quasiparticle lifetimes for $\mathrm{Sr}_{2} \mathrm{RhO}_{4}$. We show that Fermi surface volumes agree among the three techniques within $\sim 1 \%$ of an electron for all pockets, while quasiparticle masses exhibit a relative variation of $\sim 30 \%$. We consider these values to be characteristic for the precision that can realistically be obtained in favorable cases with these three techniques, and therefore conclude that for the oxide $\mathrm{Sr}_{2} \mathrm{RhO}_{4}$, STM, ARPES, and QO can extract the same information regarding Fermi surface and low-energy dispersion. The relevance of our study goes beyond $\mathrm{Sr}_{2} \mathrm{RhO}_{4}$ : our data suggest that apparent disagreements in the literature on cuprates do not arise from the intrinsic structural complexity of oxides but are likely a consequence of our limited understanding of materials with non-Fermi liquid electronic states and the applications of the techniques to such samples, especially ones with significant spatial inhomogeneity.

\section{METHODS}

\section{Sample preparation}

Our single-crystal samples were grown in a Crystal Systems four mirror image furnace using a flux feeding floating zone method. Dried $\mathrm{SrCO}_{3}$ and $\mathrm{Rh}_{2} \mathrm{O}_{3}(3 \mathrm{~N})$ were ground together in a 1:0.575 ratio, pelletised, and calcined at $1000^{\circ} \mathrm{C}$ in flowing $\mathrm{O}_{2}$ atmosphere for $24 \mathrm{~h}$. Rods were hydrostatically pressed using the usual methods and sintered at $1100^{\circ} \mathrm{C}$ for $2 \mathrm{~h}$ in flowing $\mathrm{O}_{2}$. The growth conditions in the image furnace were $100 \% \mathrm{O}_{2}$ gas at 10 bar pressure, growth speed of $10 \mathrm{mmhr}^{-1}$ and a counter rotation of $30 \mathrm{rpm}$. Subsequently, the crystals were annealed $1150^{\circ} \mathrm{C}$ under flowing oxygen for 2 weeks, as described elsewhere ${ }^{22}$.

The surfaces studied by STM and ARPES have been obtained by cleavage in ultra-high vacuum.

\section{Quantum oscillations}

Quantum oscillations measure low-energy characteristics of the electron fluid in an applied magnetic field. The oscillations, caused by the Landau quantization from the magnetic field, give precise information on the size of the Fermi pockets and the effective masses of the electrons. Quantum oscillations are a true bulk probe that is generally not influenced by surface effects but they are very sensitive to disorder in the crystals and require highquality samples to be observed. Furthermore, they also require high magnetic field and low temperatures to suppress the quasiparticle-quasiparticle scattering and the interpretation is not always simple as little information is given about the loci, shape, and type (electron or hole) of the Fermi pockets. When a strong magnetic field $\mathbf{B}$ is applied to the sample, the Landau quantization of quasiparticle orbits leads to an oscillation of the density of states at the Fermi level, periodic in reciprocal field. These oscillations are reflected in most of the physical properties; in the case of magnetoresistance they are called Shubnikov-de Haas $(\mathrm{SdH})$ oscillations ${ }^{41,52}$. By analyzing the frequency $f$ (in Tesla) of the oscillations across an inverse field range, the number and sizes of the Fermi surface pockets can be obtained. Moreover, the effective masses for the various pockets can be deduced from the temperature dependence of the oscillation amplitude (Fig. S4) via the Lifshitz-Kosevich formula, although, we note that the data analysis can be non-standard when measuring across a broad magnetic field range (for a comprehensive discussion see ref. ${ }^{22}$ ). The $\mathrm{QO}$ amplitudes also contain the Dingle temperature. These can be used to find mean free paths of $500 \AA$ for the a pocket, $714 \AA$ for the $\beta_{M}$ pocket, and $481 \AA$ for the $\beta_{x}$ pocket.

Quantum oscillation data was acquired using a standard four probe technique in a dilution refrigerator (current $I=300 \mu \mathrm{A}$ ) for temperatures between 0.1 and $1.0 \mathrm{~K}$ and magnetic fields between 7 and $15 \mathrm{~T}$. Low contact-resistance electrical connections were made to the crystals using gold wire ( 25 micron) and Dupont 6838 high-temperature curing paint (annealed at $470^{\circ} \mathrm{C}$ under $\mathrm{O}_{2}$ ). The current was applied in the $a b$ plane (the two-dimensional morphology of the crystals allowed for easy identification of the crystallographic $a b$ plane and c-axis). In the dilution refrigerator the samples were mounted on an in situ single-axis rotator for the angular quantum oscillation study. Three crystals were measured from the same batch, with consistent results.

\section{ARPES}

ARPES measures single particle excitations directly in momentum space. The most commonly used expression for the photocurrent $I(\mathbf{k}, \omega)$ is:

$I_{\text {ARPES }}=I_{0}\left|M_{f, i}\right|^{2} f(\omega) A(\mathbf{k}, \omega) * R(\delta \mathbf{k}, \delta \omega)$

where $M_{f, i}$ represents the photoemission matrix elements, $A(\mathbf{k}, \omega)$ is the spectral function, and $f(\omega)$ the Fermi function ${ }^{10}$. The expression for the intrinsic photocurrent is then convolved with the experimental momentum and energy resolution $R(\delta \mathbf{k}, \delta \omega)$. Besides experimental difficulties, complications can arise from the interference of photocurrents from different emission sites and/or from different terms in the light-matter interaction Hamiltonian. Expressing the photocurrent in terms of the spectral function further relies on the sudden approximation, i.e., the assumption that the photoexcitation is instantaneous and that there is no interaction between photoelectron and the sample during the photoemission process ${ }^{10}$. This approximation is well tested down to much lower photon energies than used in the present work.

The ARPES experiments reported in this paper have been performed at beamline 105 of Diamond Light source using photon energies in the range of $20-80 \mathrm{eV}^{53}$. Energy and momentum resolutions were set to $\sim 5 \mathrm{meV}$ / $0.008 \AA^{-1}$, except for the data shown in Fig. $2 c$ where the resolution varied with photon energy and thus with $k_{\mathrm{z}}$. All data were acquired at $\mathrm{T} \sim 8 \mathrm{~K}$.

\section{STM}

STM measures the tunneling current generated between an atomically sharp tip and a conducting sample when a voltage $V$ is applied between the two. By scanning the tip over the sample surface, STM directly delivers real-space information with atomic resolution ${ }^{54}$. The tunneling current $l$ is directly proportional to the integrated local density of states (LDOS) of quasiparticles, which in the formalism of many-body physics can be defined via the local spectral function $A(\omega, \boldsymbol{r})=\sum_{\boldsymbol{k}} A(\boldsymbol{k}, \omega ; \boldsymbol{r})$. The local spectral function of the sample can be accessed for both occupied and unoccupied states by measuring the local differential conductance ${ }^{52}$ :

$\frac{\mathrm{d} l}{\mathrm{~d} V}(e V, \mathbf{r})=\frac{4 \pi e^{2}}{\hbar}|t(\mathbf{r})|^{2} A_{\top} A_{\mathrm{S}}(\omega=e V, \mathbf{r})$

where $A_{S, T}$ are the spectral functions of sample and tip, respectively, and where we approximated the Fermi-Dirac distribution as a step function. $|t(\mathbf{r})|^{2}$ represents the position-dependent tunneling matrix element that contains the exponential dependence on tip-sample distance. Usually, the spectral function of the tip, $A_{T}$ is designed to be constant, and the momentum dependence of the tunneling matrix elements is ignored.

When measuring in spectroscopic-imaging mode (SI-STM), for each pixel on a chosen field of view a $\mathrm{d} / / \mathrm{d} V$ spectrum is acquired at the tip-sample distance determined locally by the set-up conditions $\left(V_{S}, I_{S}\right)$. The result of such a measurement is a three-dimensional dataset representing the local density of states as function of position and energy.

Because we determine the tip-sample distance at each point by the setup conditions, the effect of the matrix element (assuming it is energy independent) is canceled. However, the procedure does bring in an extra denominator: $\frac{\mathrm{d} l}{\mathrm{~d} V}(e V, \mathbf{r})=\frac{I_{s_{\mathrm{S}} A_{\mathrm{S}}(e V, \mathbf{r})}}{\int_{0}^{V_{5}} A(E, \mathbf{r}) \mathrm{d} E}(9)$. The procedure can thus introduce additional artifacts into the measured differential conductance $d / / d V^{28}$, the so-called set-up effect.

A common way to reduce this effect is to choose set-up conditions far away from the Fermi level such that inhomogeneities in the integrated density of states average out; however, this is not always experimentally possible. Other methods include the use of the ratio between quantities with positive and negative bias ${ }^{55}$, or the division of the differential conductance by the total conductance $(\mathrm{d} l / \mathrm{d} V) /(I / V)^{28-30}$ - the approach that we also use in this paper. See Fig. 5b, $c$ and Supplementary Figs 1 and 6 for comparisons. The current $I$ is the measured current at that particular location and bias $V$, which means it is small but generally non-zero at the Fermi level. The voltage $V$ is a value set in the experiment, implying for the Fermi level that the data would be multiplied by 0 in the normalization. To circumvent this, we manually add a $10 \mathrm{uV}$ (negligible to the energy scale set by temperature) offset in data processing.

The STM experiments reported in this paper have been performed with an ultra-high vacuum, home-built STM with exceptional stability, described elsewhere ${ }^{56}$. All data was taken at a base temperature of $4.2 \mathrm{~K}$. Measurements are performed with a chemically etched tungsten tip that is prepared by field emission on a gold surface before measuring $\mathrm{Sr}_{2} \mathrm{RhO}_{4}$. 


\section{DATA AVAILABILITY}

The data used in this paper is available at https://doi.org/10.26037/yareta: upufx3qatvbsldxstyz6tezszi.

\section{CODE AVAILABILITY}

The code used in this work are available from the corresponding author upon reasonable request.

Received: 5 June 2020; Accepted: 9 November 2020;

Published online: 08 December 2020

\section{REFERENCES}

1. Keimer, B., Kivelson, S. A., Norman, M. R., Uchida, S. \& Zaanen, J. From quantum matter to high-temperature superconductivity in copper oxides. Nature $\mathbf{5 1 8}$ 179-186 (2015).

2. Johnston, D. C. The puzzle of high temperature superconductivity in layered iron pnictides and chalcogenides. Adv. Phys. 59, 803-1061 (2010).

3. Coleman, P. Handbook of Magnetism and Advanced Magnetic Materials (John Wiley and Sons, 2007).

4. Imada, M., Fujimori, A. \& Tokura, Y. Metal-insulator transitions. Rev. Mod. Phys. 70, 1039-1263 (1998).

5. Sachdev, S. Quantum Phase Transitions (Cambridge Univ. Press, Cambridge, 2011).

6. Crommie, M. F., Lutz, C. P. \& Eigler, D. M. Imaging standing waves in a twodimensional electron gas. Nature 363, 524-527 (1993).

7. Petersen, L., Hofmann, P. H., Plummer, E. W. \& Besenbacher, F. Fourier transform-STM: determining the surface Fermi contour. J. Electron Spectrosc. Relat. Phenom. 109, 97-115 (2000).

8. Fujita, K. et al. in Theoretical Methods for Strongly Correlated Systems Ch. 3 (Springer, Berlin, Heidelberg, 2012).

9. Yazdani, A., Eduardo, H., Neto, S. \& Aynajian, P. Spectroscopic imaging of strongly correlated electronic states. Annu. Rev. Condens. Matter Phys. 7, 11 (2016).

10. Hüfner, S. Photoelectron Spectroscopy (Springer, Berlin, Heidelberg, 2003).

11. Baumberger, F. et al. Fermi surface and quasiparticle excitations of $\mathrm{Sr}_{2} \mathrm{RhO}_{4}$. Phys. Rev. Lett. 96, 246402 (2006)

12. Coleman, P. Introduction to Many-Body Physics (Cambridge Univ. Press, Cambridge, 2013).

13. Damascelli, A., Hussain, Z. \& Shen, Z. Angle-resolved photoemission studies of the cuprate superconductors. Rev. Mod. Phys. 75, 473-541 (2003).

14. Sebastian, S. E. \& Proust, C. Quantum oscillations in hole-doped cuprates. Annu. Rev. Condens. Matter Phys. 6, 411-430 (2015).

15. McElroy, K. et al. Elastic scattering susceptibility of the high temperature superconductor $\mathrm{Bi}_{2} \mathrm{Sr}_{2} \mathrm{CaCu}_{2} \mathrm{O}_{8+\delta}$ : a comparison between real and momentum space photoemission spectroscopies. Phys. Rev. Lett. 96, 067005 (2006).

16. Markiewicz, R. S. Bridging $\mathrm{k}$ and $\mathrm{q}$ space in the cuprates: comparing angleresolved photoemission and STM results. Phys. Rev. B 69, 1-10 (2004)

17. Vishik, I. M. et al. A momentum-dependent perspective on quasiparticle interference in $\mathrm{Bi}_{2} \mathrm{Sr}_{2} \mathrm{CaCu}_{2} \mathrm{O}_{8+\delta}$. Nat. Phys. 5, 718-721 (2009).

18. Hashimoto, $M$. et al. Reaffirming the $d$-wave superconducting gap using the autocorrelation angle-resolved photoemission spectroscopy. Phys. Rev. Lett. 106, 167003 (2011).

19. Kirchner, S. et al. Heavy-electron quantum criticality and single-particle spectroscopy. Rev. Mod. Phys. 92, 011002 (2020).

20. Matt, C. E. et al. Consistency between ARPES and STM measurements on $\mathrm{SmB}_{6}$ Phys. Rev. B 101, 085142 (2020).

21. Kim, B. J. et al. Missing $x y$-band Fermi surface in $4 \mathrm{~d}$ transition-metal oxide $\mathrm{Sr}_{2} \mathrm{RhO}_{4}$ : effect of the octahedra rotation on the electronic structure. Phys. Rev. Lett. 97, 106401 (2006).

22. Perry, R. S. et al. $\mathrm{Sr}_{2} \mathrm{RhO}_{4}$ : a new, clean correlated electron metal. N. J. Phys. 8 , 175-175 (2006)

23. Baumberger, F. et al. Nested Fermi surface and electronic instability in $\mathrm{Ca}_{3} \mathrm{Ru}_{2} \mathrm{O}_{7}$ Phys. Rev. Lett. 96, 107601 (2006).

24. Haverkort, M. W., Elfimov, I. S., Tjeng, L. H., Sawatzky, G. A. \& Damascelli, A. Strong spin-orbit coupling effects on the Fermi surface of $\mathrm{Sr}_{2} \mathrm{RuO}_{4}$ and $\mathrm{Sr}_{2} \mathrm{RhO}_{4}$. Phys. Rev. Lett. 101, 26406 (2008).

25. Liu, G., Antonov, V. N., Jepsen, O. \& Andersen., O. K. Coulomb-enhanced spin-orbit splitting: the missing piece in the $\mathrm{Sr}_{2} \mathrm{RhO}_{4}$ puzzle. Phys. Rev. Lett. 101, 026408 (2008).

26. Kim, M., Mravlje, J., Ferrero, M., Parcollet, O. \& Georges, A. Spin-orbit coupling and electronic correlations in $\mathrm{Sr}_{2} \mathrm{RuO}_{4}$. Phys. Rev. Lett. 120, 126401 (2018).

27. Tamai, A. et al. High-resolution photoemission on $\mathrm{Sr}_{2} \mathrm{RuO}_{4}$ reveals correlationenhanced effective spin-orbit coupling and dominantly local self-energies. Phys. Rev. X 9, 021048 (2019)
28. Feenstra, R. M., Stroscio, J. A. \& Fein, A. P. Tunneling spectroscopy of the Si(111) 2x1 surface. J. Vac. Sci. Technol. B 5, 295-306 (1987).

29. Hanaguri, T. et al. Two distinct superconducting pairing states divided by the nematic end point in $\mathrm{FeSe}_{1-x} \mathrm{~S}_{x}$. Sci. Adv. 4, 6419 (2018)

30. Kostin, A. et al. Imaging orbital-selective quasiparticles in the Hund's metal state of FeSe. Nat. Mater. 17, 869-874 (2018).

31. Macdonald, A. J. et al. Dispersing artifacts in FT-STS: a comparison of set point effects across acquisition modes. Nanotechnology 27, 414004 (2016).

32. Allan, M. P. et al. Imaging Cooper pairing of heavy fermions in CeColn ${ }_{5}$. Nat. Phys. 9, 468-473 (2013).

33. Wang, Z. et al. Quasiparticle interference and strong electron-mode coupling in the quasi-one-dimensional bands of $\mathrm{Sr}_{2} \mathrm{RuO}_{4}$. Nat. Phys. 13, 799-805 (2017).

34. Allan, M. P. et al. Anisotropic energy gaps of iron-based superconductivity from intraband quasiparticle interference in LiFeAs. Science 336, 563-567 (2012).

35. Hess, C. et al. Interband quasiparticle scattering in superconducting LiFeAs reconciles photoemission and tunneling measurements. Phys. Rev. Lett. 110, 017006 (2013).

36. Wang, Q. \& Lee, D. Quasiparticle scattering interference in high-temperature superconductors. Phys. Rev. B 67, 020511 (2003).

37. Capriotti, L., Scalapino, D. J. \& Sedgewick, R. D. Wave-vector power spectrum of the local tunneling density of states: ripples in a $d$-wave sea. Phys. Rev. $B \mathbf{6 8}$ 014508 (2003).

38. Nunner, T. S., Chen, W., Andersen, B. M., Melikyan, A. \& Hirschfeld, P. J. Fourier transform spectroscopy of $d$-wave quasiparticles in the presence of atomic scale pairing disorder. Phys. Rev. B 73, 1-7 (2006).

39. Sulangi, M. A., Allan, M. P. \& Zaanen, J. Revisiting quasiparticle scattering interference in high-temperature superconductors: the problem of narrow peaks. Phys. Rev. B 96, 134507 (2017).

40. Choubey, P., Kreisel, A., Berlijn, T., Andersen, B. M. \& Hirschfeld, P. J. Universality of scanning tunneling microscopy in cuprate superconductors. Phys. Rev. B 96, 174523 (2017).

41. Schoenberg, D. Magnetic Oscillations in Metals (Cambridge Univ. Press, Cambridge, 1984).

42. Bürgi, L., Jeandupeux, O., Brune, H. \& Kern, K. Probing hot-electron dynamics at surfaces with a cold scanning tunneling microscope. Phys. Rev. Lett. 82, 4516 (1999).

43. Allan, M. P. et al. Identifying the 'fingerprint' of antiferromagnetic spin fluctuations in iron pnictide superconductors. Nat. Phys. 11, 177-182 (2014).

44. Dahm, T. \& Scalapino, D. J. Quasi-particle interference probe of the self-energy. $N$. J. Phys. 16, 023003 (2014).

45. Sulangi, M. A. \& Zaanen, J. Self-energies and quasiparticle scattering interference. Phys. Rev. B 98, 094518 (2018).

46. Martins, C., Aichhorn, M., Vaugier, L. \& Biermann, S. Reduced effective spin-orbital degeneracy and spin-orbital ordering in paramagnetic transition-metal oxides: $\mathrm{Sr}_{2} \mathrm{IrO}_{4}$ versus $\mathrm{Sr}_{2} \mathrm{RhO}_{4}$. Phys. Rev. Lett. 107, 266404 (2011).

47. Martins, C. et al. Coulomb correlations in $4 \mathrm{~d}$ and $5 \mathrm{~d}$ oxides from first principles or how spin-orbit materials choose their effective orbital degeneracies. J. Phys. 29, 263001 (2017).

48. Chaplik, A. V. Energy spectrum and electron scattering processes in inversion layers. Sov. J. Exp. Theor. Phys. 33, 997 (1971).

49. Giuliani, G. F. \& Quinn, J. J. Lifetime of a quasiparticle in a two-dimensional electron gas. Phys. Rev. B 26, 4421 (1982).

50. Fukuyama, H. \& Abrahams, E. Inelastic scattering time in two-dimensional disordered metals. Phys. Rev. B 27, 5976 (1983).

51. Smith, N. V., Thiry, P. \& Petroff, Y. Photoemission linewidths and quasiparticle lifetimes. Phys. Rev. B 47, 15476 (1993).

52. Bergemann, C., Mackenzie, A. P., Julian, S. R., Forsythe, D. \& Ohmichi, E. Quasi-twodimensional Fermi liquid properties of the unconventional superconductor $\mathrm{Sr}_{2} \mathrm{RuO}_{4}$. Adv. Phys. 52, 639-725 (2003).

53. Hoesch, M. et al. A facility for the analysis of the electronic structures of solids and their surfaces by synchrotron radiation photoelectron spectroscopy. Rev. Sci. Instrum. 88, 013106 (2017).

54. Chen, C. J. Introduction to Scanning Tunneling Microscopy (Oxford Univ. Press, Oxford, 2007).

55. Kohsaka, Y. et al. An intrinsic bond-centered electronic glass with unidirectional domains in underdoped cuprates. Science 315, 1380-1385 (2007).

56. Battisti, I., Verdoes, G., van Oosten, K., Bastiaans, K. M. \& Allan, M. P. Definition of design guidelines, construction, and performance of an ultra-stable scanning tunneling microscope for spectroscopic imaging. Rev. Sci. Instrum. 89, 123705 (2018).

\section{ACKNOWLEDGEMENTS}

This work was supported by the UK-EPSRC under grant EP/G007357/1, by the Swiss National Science Foundation (SNSF) under grants 200020_165791 and 200020_184998, 
by the Max Planck Society, by the European Research Council (ERC StG SpinMelt), and by the Netherlands Organization for Scientific Research (NWO) under grants 680-47-536 and FOM-167. We acknowledge Diamond Light Source for time on beamline 105 under proposals no. SI13398 and SI5282.

\section{AUTHOR CONTRIBUTIONS}

I.B., W.T., and A.T. performed STM experiments; A.T. and S.R. performed ARPES experiments; R.P. performed QO experiments and fabricated samples. All authors participated in discussion, data analysis, and interpretation.

\section{COMPETING INTERESTS}

The authors declare no competing interests.

\section{ADDITIONAL INFORMATION}

Supplementary information is available for this paper at https://doi.org/10.1038/ s41535-020-00292-4.

Correspondence and requests for materials should be addressed to A.P.M., F.B. or M.P.A.
Reprints and permission information is available at http://www.nature.com/ reprints

Publisher's note Springer Nature remains neutral with regard to jurisdictional claims in published maps and institutional affiliations.

(c) (i)

Open Access This article is licensed under a Creative Commons Attribution 4.0 International License, which permits use, sharing, adaptation, distribution and reproduction in any medium or format, as long as you give appropriate credit to the original author(s) and the source, provide a link to the Creative Commons license, and indicate if changes were made. The images or other third party material in this article are included in the article's Creative Commons license, unless indicated otherwise in a credit line to the material. If material is not included in the article's Creative Commons license and your intended use is not permitted by statutory regulation or exceeds the permitted use, you will need to obtain permission directly from the copyright holder. To view a copy of this license, visit http://creativecommons. org/licenses/by/4.0/.

(c) The Author(s) 2020 